\title{
13
}

\section{Topography and Dynamics of the Olfactory System}

\author{
S. SACHSE ${ }^{1}$ and C. G. GALIZIA ${ }^{2}$ \\ ${ }^{1}$ Laboratory of Neurogenetics and Behavior, The Rockefeller University, \\ New York, NY 10021, U.S.A. \\ ${ }^{2}$ Dept. of Entomology, University of California at Riverside, \\ Riverside, CA 92521, U.S.A.
}

\begin{abstract}
The chemical senses - taste and smell - are evolutionarily the most ancient animal senses. They are characterized by a multidimensional and diverse stimulus space, consisting of many odorous molecules that cannot be classified along any small set of dimensions. Animals detect these odors with specialized olfactory sensory neurons that express one or a few ligand-binding odorant receptor (OR) proteins. Animals cope with the problem of recognizing an extremely large number of different odorants by programming a very large number of functionally different olfactory neurons. Odors activate these neurons and generate characteristic activity patterns across the population of these receptors, which are relayed to second-order olfactory neurons. The entire available raw information about the animal's olfactory environment is present in these patterns; however, olfactory information is further processed before it is relayed to higher-order brain centers. This chapter reviews the role played by individual neurons in microcircuits in the first olfactory synapse in modulating this information. How is information extracted about odor quality, odor concentration, and scent components? Identity, spatial, and temporal coding mechanisms are discussed.
\end{abstract}

\section{INTRODUCTION}

Microcircuits in the antennal lobe optimize olfactory coding. Such a statement clearly requires qualification. All animals with an advanced olfactory system have an "olfactory lobe" (the insect antennal lobe or the vertebrate olfactory bulb), in which axons from olfactory sensory neurons converge onto discrete, spherical structures - the olfactory glomeruli-local neurons make lateral connections, and output neurons relay the processed activity to higher-order 
brain centers. "Olfactory coding" means transforming information about an olfactory stimulus into patterns of neuronal activity so that subsequent processing steps have access to that information. In that sense finding the "code" is equivalent to understanding the "language" of the brain. The code differs along the processing steps leading from the receptor neurons to a behavioral decision. For example, olfactory information is fully "encoded" within the sensory organs by the receptor neurons in the periphery, since all information about an olfactory stimulus that is available to the animal is in that activity pattern. Similarly, it is fully "encoded" within the olfactory lobe; the coding principles are, however, different and not yet fully understood. It is also fully "encoded" in the output neurons of the antennal lobe, most likely according to different coding principles. "Optimization" indicates that the code is improved along these steps. For example, coding is optimized when information relevant to the animal is enhanced with respect to less relevant content, when signal is increased over noise, when sensitivity is increased, and/or when the coding scheme is "reformatted" in a way that makes it easier and more reliable to decode so that information is better extracted. Such reformatting may allow information to be stored more economically and to be more suitable for memory formation, which would lead to an improved retrieval from memory. Such information could be the identity of an odor stimulus (an identification task), its concentration (a measurement along a continuous variable), or the identity of odor components in a mixture (an analytical task).

We consider the mechanisms that insect antennal lobes employ to optimize their coding capacity. Recent studies on insect olfaction have provided important insights into the function of the antennal lobe. Several controversies in the field remain to be settled, such as the significance of spatial versus temporal features, or reports that incoming activity patterns are not processed at this early stage (see below). Here we argue that the antennal lobe is actively involved in extracting relevant information from the sensory input, and that this process is accomplished by a set of several local neuron networks. We strengthen the notion of olfactory glomeruli as the functional units in this process. We propose specific working hypotheses for how odor identity and stimulus concentration are encoded, and how odor mixture analysis and high sensitivity are achieved.

\section{BACKGROUND INFORMATION}

We begin with a brief description of the neurons in the insect antennal lobe and then draw comparisons between different insect species as well as to vertebrates. We then review physiological properties. Discussion about the significance of morphological and physiological findings for olfactory coding is discussed thereafter. 


\section{Morphological Elements of the Insect Olfactory System}

\section{The Cells in the Insect Antennal Lobe}

Odors are recognized by primary olfactory sensory neurons (OSNs), which are located on the insect antenna and express odorant receptor genes that encode the odorant receptors (ORs). These are seven transmembrane domain receptor proteins that interact with odor molecules and transduce odorant binding to cellular excitation. The organization of the insect olfactory system is shown in Figure 13.1, using the honeybee as an example. The OSNs send their axons to the olfactory neuropil, the insect antennal lobe (Figure 13.1a, b), which consists of discrete neuropil structures called olfactory glomeruli. Each OSN expresses a single OR gene, and all OSNs expressing the same OR converge onto a common glomerulus. A glomerulus collects OSNs of only one type. Thus, the glomerulus acts as a "collecting basket" of OSNs with similar odor response profiles. This correspondence has been shown for Drosophila melanogaster (Vosshall 2001) and is also assumed for other insect species, but has not yet been experimentally proven. However, a few cases of 1:2 and of 2:1 innervation ratios in $D$. melanogaster have been described as well. A glomerulus receives not only the input from OSNs, but contains a highly ordered synaptic organization including synaptic microcircuits among OSNs, local interneurons, and projection neurons. The cell bodies of local interneurons and projection neurons are located outside of the glomeruli, in the periphery of the antennal lobe. Local interneurons branch exclusively within the antennal lobe and, in honeybees, can be further divided into two classes (Figure 13.1c): homogeneous local interneurons globally innervate many glomeruli, whereas heterogeneous local interneurons innervate densely a single glomerulus and diffusely a few others (Fonta et al. 1993). Projection neurons represent the antennal lobe output neurons and relay the olfactory information to higher processing centers, such as the lateral protocerebrum and the mushroom bodies. Projection neurons are either uniglomerular neurons, and thus collect synaptic input in just one glomerulus, or multiglomerular neurons (Figure 13.1d). Uniglomerular projection neuron axons innervate both the lateral protocerebrum and the mushroom bodies, where they diverge onto many Kenyon cells, which in turn are read out by mushroom body extrinsic neurons. Multiglomerular projection neurons send their axons only to the lateral protocerebrum and around the $\alpha$-lobe, bypassing the mushroom bodies. However, projection neurons in honeybees also have small branches at their exit points of the antennal lobe, which may form connections between cells outside their innervated glomeruli (Müller et al. 2002). The computational function of these lateral pathways is not yet understood.

Distinct classes of neurotransmitters mediate communication between different neuron types. OSNs release acetylcholine to excite local interneurons or projection neurons (Homberg and Müller 1999). Local interneurons are mainly inhibitory and use GABA and/or histamine to inhibit projection neurons, other 
(a)

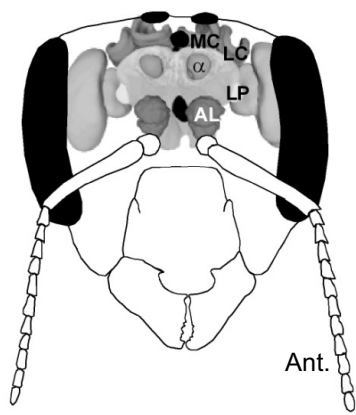

(c)

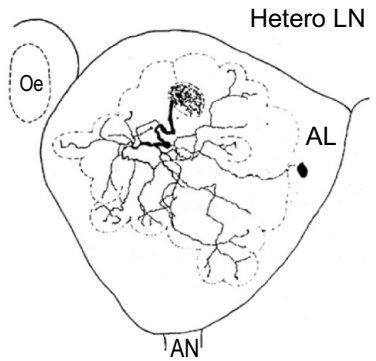

(d)

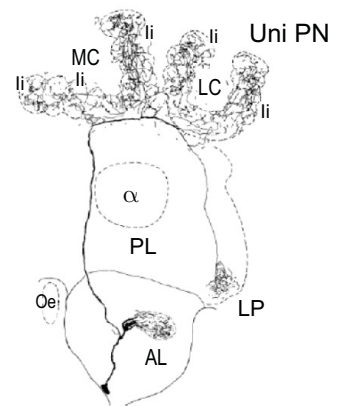

(b)
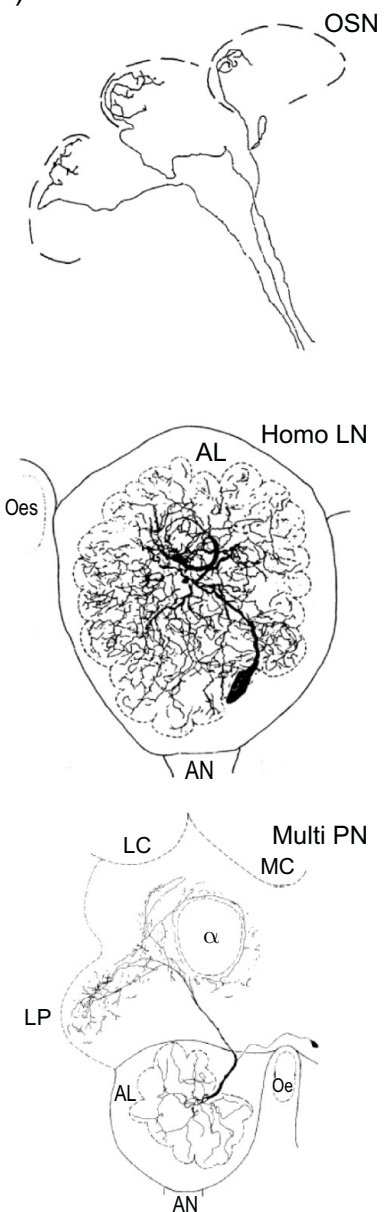

Figure 13.1 Morphology of olfactory neurons in the honeybee Apis mellifera. (a) Schematic view of the honeybee brain. The olfactory pathway leads from the antenna (Ant.) to the first olfactory neuropil, the antennal lobe (AL). The projection neurons (PNs) send their axons to higher processing centers: the lateral protocerebrum (LP) and the medial and lateral mushroom body (MB) calyx (MC and LC). $\alpha$ : $\alpha$-lobe of the MB. (b) Reconstruction of three olfactory sensory neurons (OSNs). Each OSN innervates the rind of a single glomerulus in the AL (adapted from Brockmann and Brückner 1995). (c) Reconstruction of two different morphological types of local interneurons (LNs) in the honeybee AL. Heterogeneous LNs (hetero LNs) densely innervate a single glomerulus and sparsely a few others, while homogeneous LNs (homo LNs) diffusely innervate up to 100 glomeruli (adapted from Abel 1997). (d) Branching pattern of a uniglomerular (uni $\mathrm{PN}$ ) and a multiglomerular projection neuron (multi PN) in the honeybee brain. Uniglomerular PNs collect the olfactory information of a single glomerulus in the AL and send their axons to the MB (medial and lateral calyx, MC, LC) and the LP. Multiglomerular PNs innervate many glomeruli and relay the information to the LP (adapted from Abel 1997). $\mathrm{Oe}=$ oesophagus; $\mathrm{PL}=$ posterior lobe. 
local interneurons, or OSNs via a feedback loop. A subgroup of uniglomerular projection neurons uses acetylcholine, the transmitter of a second subgroup is as yet unknown, and at least some multiglomerular projection neurons release GABA.

\section{Differences between Insect Species}

Despite striking similarities among insects (and, in fact, between insects and vertebrates), there are many important differences (Figure 13.2): convergence ratios between OSNs and projection neurons, numbers of units, innervation logic of glomerular microcircuits, morphology of cells, and transmitters used.

For example, a single honeybee glomerulus is innervated on average by almost 400 OSNs (60,000 OSNs onto 160 glomeruli), 1000 local interneurons

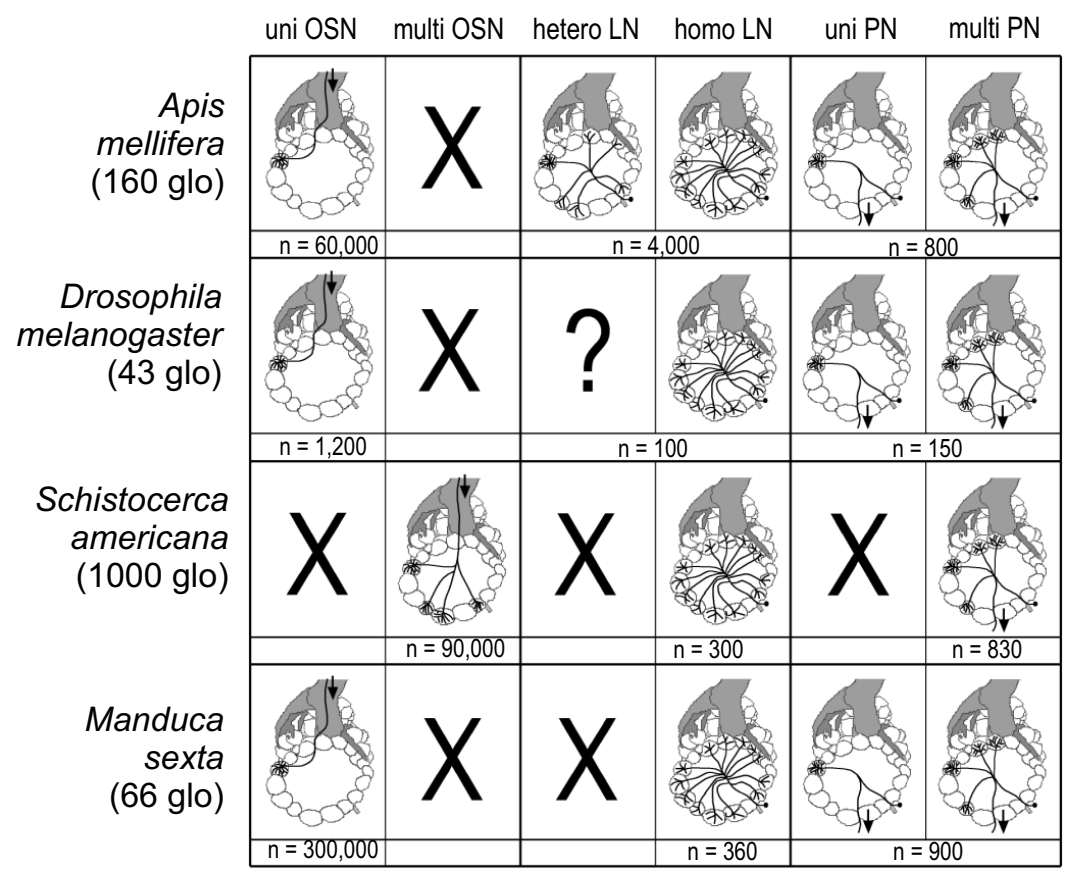

Figure 13.2 Olfactory sensory neurons (OSNs) in different insect species. The number of glomeruli (glo), neurons (n), innervation ratio, and neuronal morphology is species specific. If a particular cell type has never been described for a species, that entry is marked with an "X." Heterogeneous local interneurons (LNs) may be present in Drosophila melanogaster, but their morphology has not yet been described. Numbers are approximate and have been collected from a variety of references. In addition, if no separate count is available, only the sum of two cell types is given. In bees, most LNs are hetero LNs, but in flies that may be the other way round. Most projection neurons (PNs) are uniglomerular in bees, flies, and moths. It is unclear whether a more complex organization, per se, affords a more sophisticated behavioral spectrum. 
(assuming a total of 4000 local interneurons and that each local interneuron innervates an average of 40 glomeruli), and 5 projection neurons ( 800 projection neurons in 160 glomeruli). The fruit fly $D$. melanogaster has many fewer OSNs and, therefore, a ratio between OSN and glomeruli of only 30:1. However, since most OSNs project into the antennal lobes of both sides, the average count of OSNs in each glomerulus is 50 in this species.

In contrast to other insects, locusts have only multiglomerular projection neurons. Similarly, individual OSN axons innervate several glomeruli. The glomerular groups innervated by projection neurons and OSNs do not coincide, suggesting that the multiglomerular organization is not a mere split of glomeruli into sub-glomeruli, but rather reflects a qualitatively different architecture. Indeed, locust antennal lobes consists of about 1000 "mini" glomeruli with different cytoarchitecture and innervation patterns. Therefore, in this species glomeruli either do not form functional units or the function of these units differs from that in other insects.

In addition to architectural differences, neurotransmitters also differ between insect species. For example, the occurrence of histaminergic local interneurons is not ubiquitous to insects. Apart from honeybees, only cockroaches and crickets possess histaminergic olfactory cells (Nässel 1999). The antennal lobes of moths, locusts, and dipteran flies lack histamine immunoreactivity. The species-specific function of histaminergic local interneurons, with regard to odor coding and processing, is still unknown and needs further investigation.

These species-specific differences show the obvious but often overlooked fact that generalizations among insect species have to be made with caution. Many features are shared, but each species is unique, and it may not always be possible to extrapolate observations made in one species to other species.

\section{Comparison to the Vertebrate Olfactory Bulb}

The olfactory bulb, which represents the analogue to the insect antennal lobe, displays important similarities in terms of anatomical and functional features (Figure 13.3). Both are spherical structures and consist of olfactory glomeruli. Current evidence suggests that, as in insects, OSNs expressing a given OR in mice project to a restricted number of topographically fixed glomeruli. Moreover, the vertebrate olfactory system has direct cellular counterparts for many of the cell types just discussed in insects: OSNs reside in the nasal epithelium and are morphologically similar to insect OSNs, mitral/tufted cells correspond to insect projection neurons, whereas granule cells and periglomerular cells are likely the functional relatives of insect local interneurons. Mitral/tufted cells are uniglomerular in rodents, but multiglomerular in zebra fish. Claiming a more detailed match (e.g., between heterogeneous local interneurons and homogeneous local interneurons with particular olfactory bulb interneurons) is inappropriate. One important anatomical difference between both systems is their layering: although all synapses of the antennal lobe are in the glomeruli, in the 
Insect AL

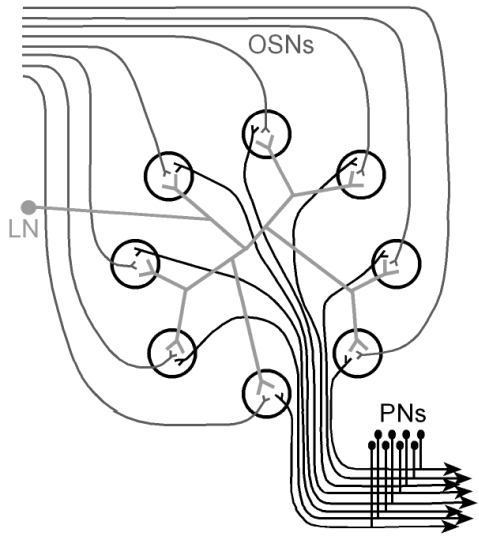

Vertebrate OB

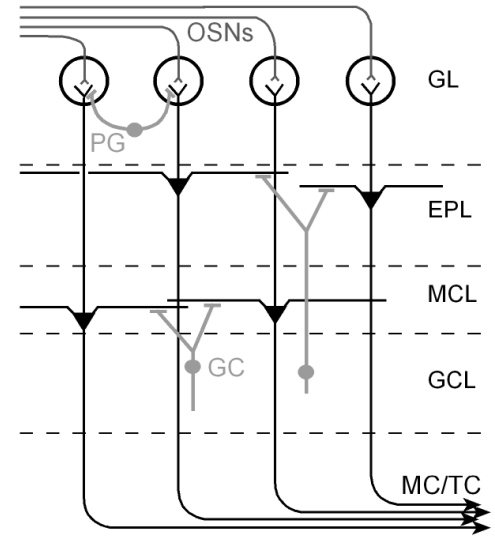

Figure 13.3 Organization of the insect antennal lobe (AL) versus the vertebrate olfactory bulb $(\mathrm{OB})$. The olfactory neurons in the vertebrate OB represent counterparts of olfactory neurons in the insect AL. The OB, however, is organized in distinct layers, in which different processing steps take place, whereas in the AL, synaptic interactions only appear within a glomerulus. (OSN, olfactory sensory neuron; LN, local interneuron; PN, projection neuron; PG, periglomerular cell; GC, granule cell; MC, mitral cell; TC, tufted cell; GL, glomerular layer; EPL, external plexiform layer; MCL, mitral cell layer; GCL, granule cell layer). Adapted from Hildebrand and Shepherd (1997).

olfactory bulb, the glomerular layer is but one of several layers. In insects, projection neurons interact synaptically with local interneurons and OSNs almost exclusively within glomeruli. Their vertebrate counterparts show many synaptic contacts in deeper layers of the olfactory bulb distant from glomeruli.

\section{Other Olfactory Systems}

Specialized olfactory structures exist to process information about special stimuli, such as pheromones, which are used for intraspecific communication. These structures do not always follow the anatomical organization described above, which indicates that evolution has found a variety of solutions for olfactory coding. These structures are not considered here.

\section{Physiological Responses to Odors}

\section{Responses in Olfactory Sensory Neurons}

Odors elicit combinatorial across-OSN patterns of activity. Some odors may activate only one or a few OSNs, but most will activate several. As odor concentration is increased, activation is stronger, and more OSNs are recruited. The responses of OSNs have been characterized in a variety of species, with $D$. melanogaster being the best described system to date using single-sensilla recordings (de Bruyne et al. 2001). 
Whether OSNs are narrowly or broadly tuned is a matter of vigorous debate. A system based on narrowly tuned OSNs is a labeled line system: each OSN only responds to a single substance, and thus when that OSN is active, the substance is in the environment. Such a system is very specialized, and in its extreme form, the number of odors that can be coded corresponds to the number of OSNs with different response profiles. In contrast, a system based on broadly tuned OSNs relies on combinatorial codes. Each odor activates many OSN types, and each OSN can be activated by many odors. It is only by analyzing the combination of active glomeruli that the animal can identify an odor. Many OSNs respond to a variety of substances at high concentration, but may be exquisitely sensitive to a few molecules at a concentration several orders of magnitude lower. Should we regard these as examples of a highly sensitive labeled line system, or rather as units in a combinatorial code? The answer lies in the ecology of the species: Even the most prominent cases of a labeled line (i.e., moth pheromone receptors) respond to chemicals other than the pheromone itself(indeed, some labs consistently use chemically more stable substitutes instead of the original pheromone component). However, such alien substances do not exist in the natural environment of these animals, and therefore a strong argument is rightly made for a labeled line system. For most other substances, however, animals are exposed to odorants over a large range of concentrations in their natural environment. A honeybee, for example, can smell an odor source over large distances (low concentration task), but can also recognize it when sitting on the flower (high concentration task). The olfactory system should therefore be capable of differentiating, for a given OSN, between the occurrence of a low-concentration best ligand or a high-concentration secondary ligand. This cannot be done on the basis of a single OSN response itself. It must be done through pattern analysis across several OSNs.

Most OSN responses are temporally structured. Some are inhibited by odors and show rebound excitation at the end of the stimulus; some fire for a long time irrespective of stimulus duration; others only fire for a very short time and even stop if the stimulus continues. Some of these effects are probably due to adaptation of the OSNs (tonic vs. phasic response properties). The consequence for the central nervous system is that it gets a temporally complex input from the OSNs. Whether these temporal structures are used for odor analysis and whether the periphery rather than the antennal lobe network may cause part of the temporal patterns observed within the antennal lobe is discussed below.

\section{Responses in Local Interneurons}

Most, or possibly all, local interneurons are inhibitory. Many are spontaneously active and respond to odors. In honeybees there is a prominent population of heterogeneous local interneurons that have distinct odor-response profiles comparable to uniglomerular projection neurons. In some cases the odors they responded to could be shown to correspond to the odors expected from the 
functional atlas (Galizia and Kimmerle 2004). In D. melanogaster, homogeneous local interneurons show very broad response profiles, responding to most odors (Ng et al. 2002; Wilson et al. 2004). Local interneurons shape the responses in projection neurons in at least two ways: (a) with a very fast inhibition, mediated by GABA acting on ionotropic channels, and (b) with a slower timescale (Christensen et al. 1998). Studies with pharmacological antagonists have shown that the fast GABA-mediated currents cause synchrony among projection neuron spikes and odor-evoked oscillations in the range of 20-30 Hz. Local interneurons in honeybees, flies, and moths are spiking; in locusts they are non-spiking.

\section{Responses in Projection Neurons}

Responses in insect projection neurons have been measured at the single-cell level with electrophysiological techniques in a variety of species (Christensen et al. 1998; Hansson and Christensen 1999; Müller et al. 2002; Galizia and Kimmerle 2004) and with optical methods, by loading projection neurons with calcium sensitive dyes (in bees; Sachse and Galizia 2002, 2003) or by expressing activity-sensitive proteins in projection neurons only (in flies; Fiala et al. 2002; Ng et al. 2002; Wang et al. 2003). Comparing response profiles across many odors yielded somewhat contradictory results. In bees we found that the responses in projection neurons are modified with respect to the input, as estimated from bath applied dyes (Sachse and Galizia 2003), and shaped by the antennal lobe network, as shown by pharmacological manipulation (Sachse and Galizia 2002; Figure 13.4). This "reformatting" consists of the removal of weak glomeruli from the activity patterns. Therefore, whenever a glomerulus gave a strong response in the input, it also gave a strong response in the output. In contrast, other groups found in D. melanogaster that there was no difference between the OSN input and the uniglomerular projection neuron output in terms of molecular response profiles measured either by intracellular calcium increase (Wang et al. 2003) or by synaptic transmitter release (Ng et al. 2002). A recent study in D. melanogaster, however, contradicts these reports. Experiments using direct patch clamping of uniglomerular projection neurons and singlesensilla recordings of OSNs showed that uniglomerular projection neurons have broader response profiles than the OSNs that innervate the same glomerulus (Wilson et al. 2004). Because of the many differences in these studies, including different techniques, analysis methods, and experimental animals, we find that none of the studies described above is conclusive enough to draw firm conclusions about the logic of input-output relationships within olfactory glomeruli.

\section{Antennal Lobe Output and Mushroom Body Input}

What is the fate of across-projection neuron activity patterns on their way to the mushroom bodies? This question is not directly related to the antennal lobe, but 
(a)

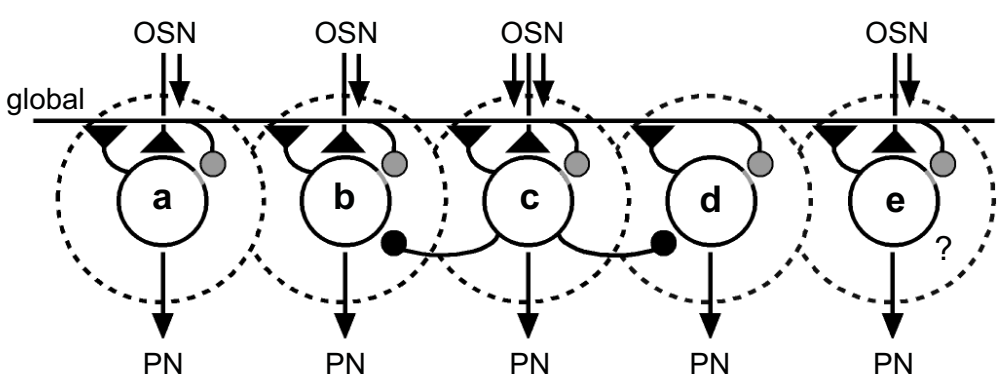

(b)

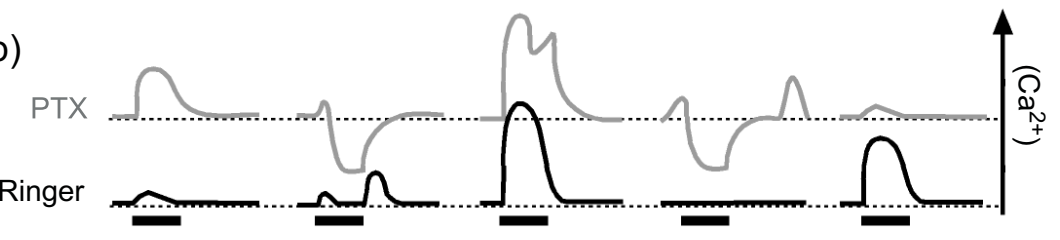

Figure 13.4 Two inhibitory networks shape the odor representation of projection neuron (PN) responses in the honeybee. (a) Model of the functional connectivity between olfactory glomeruli when stimulated with a particular odor. Glomerulus $\mathbf{c}$ gets strong OSN input and inhibits other glomeruli with picrotoxin (PTX)-insensitive synapses (black circles). Glomeruli a, b, and e get weak OSN input; $\mathbf{d}$ gets no OSN input for this odor. All glomeruli feed into a global, PTX-sensitive inhibitory network (gray circles). Thus, application of PTX leads to an increase of the PN response of weakly activated glomeruli a and a prolongation of glomeruli with a strong OSN input c. The tonic increase in intracellular calcium and spontaneous activity due to PTX leads to the calcium decrease becoming more visible; thus the inhibitory response of inhibited glomeruli is enhanced b, and inhibitory PN responses during odor stimulation are visible in previously nonresponding glomeruli $\mathbf{d}$, in particular if they are spontaneously active shortly before the stimulus onset. The reduction of type e glomeruli following PTX application may be due to PN desynchronization. (b) Examples of time courses of PN calcium responses to odors: gray indicates superfusion with PTX; black depicts Ringer control. PTX leads to increased resting $\left(\mathrm{Ca}^{2+}\right)$ levels and changes the response properties, which can be categorized as five types of effects (a-e), corresponding to the glomeruli in the model. Figure adapted from Sachse and Galizia (2002).

it is still relevant, because understanding the next step may help us to decipher the output patterns. There is a massive divergence between projection neurons and the intrinsic neurons of the mushroom bodies, the Kenyon cells: in the honeybee, from 800 projection neurons onto 180,000 Kenyon cells. Although most projection neurons have a relatively broad response profile with various levels of activity strength, in Kenyon cells a large number of units encodes that same information with very few spikes, in almost binary fashion (each Kenyon cell is either active or not within each activity cycle), with only few units active at any time (Figure 13.5; Perez-Orive et al. 2002). Such logic strongly contrasts the antennal lobe, where each projection neuron covers a high dynamic range. 


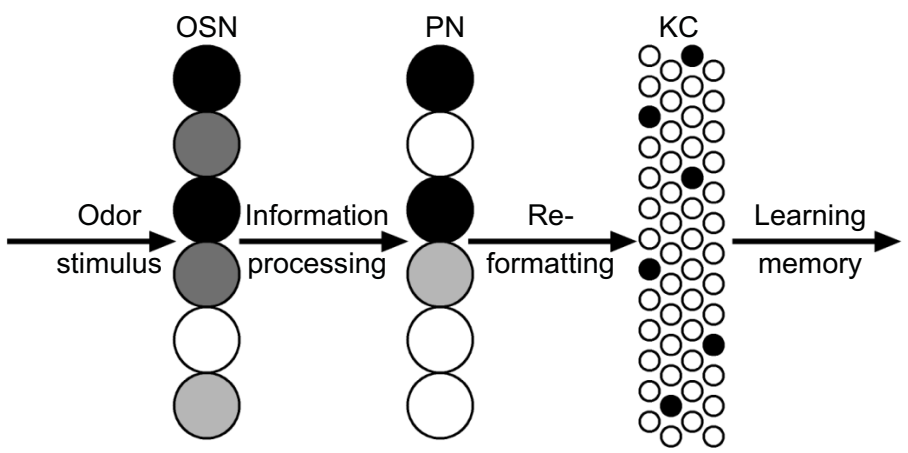

Figure 13.5 Processing levels in the antennal lobe (AL). Schematic diagram of the steps in olfactory coding. All information of an odor is necessarily coded in the combinatorial pattern of activity across receptor neurons (olfactory sensory neuron, OSN). This pattern is processed in the AL and modified into a new combinatorial pattern across projection neurons (PN). The pattern is reformatted in the mushroom body calyces onto a much larger number of Kenyon cells (KC). Note, however, the simplified nature of the figure; for example, olfactory memory traces are also found in the AL across PNs.

However, this transformation appears useful because it may facilitate the readout mechanism (Laurent 2002) and may allow more efficient learning (Heisenberg 2003).

\section{THE OLFACTORY CODE}

\section{Coding at Sequential Levels}

Looking at the olfactory system in a sequential fashion is a gross simplification, since several feedback channels influence the response patterns at all levels. Nevertheless, such an approach helps us to understand the essence of the code at each level, and all are part of "the" olfactory code. The levels to be considered here are OSNs (glomerular input), projection neurons (glomerular output), and Kenyon cells in the mushroom bodies (Figure 13.5). Olfactory sensory neurons produce a filtered and selective image of the chemical environment. It is trivial to state that all olfactory information available to the brain must be present in the combinatorial and temporally fluctuating activity patterns across the receptor neurons. It is likely that not all information present in the OSNs is processed in the antennal lobe, which would act as an additional filter for relevant information. The question is what and how information is extracted. Our view is that within the antennal lobe, the cellular network formed by OSNs, local interneurons, and projection neurons extracts information and creates an optimized odor representation, which is present at the output of the antennal lobe. The activity patterns across projection neurons are, again, combinatorial and temporally fluctuating (Figure 13.5). Finally, the massive numerical divergence 
accomplished by the transition from projection neurons to Kenyon cells is associated with a further change in coding, which will not be addressed here (Laurent 2002; Heisenberg 2003).

\section{Identity, Spatial, and Temporal Coding}

Olfactory information is necessarily coded across the activity patterns of projection neurons, but how exactly this is done is still unclear. Much discourse has focused on the issue of whether coding is spatial, identity based, or temporal. Let us review these in sequence and ask whether there is any evidence of sufficiency (i.e., this coding could work alone) or necessity (i.e., without it, coding would not work) for each.

\section{Identity Coding}

When the identity of an active neuron is relevant, we speak of an identity code. In the olfactory system, this is certainly the case. A given odor elicits specific patterns of activity in glomeruli that can be predicted. For example, in the honeybee the response profile of 38 glomeruli ( $23 \%$ of all glomeruli) has already been described for 53 odors (Galizia and Menzel 2001). The molecular basis of this finding is becoming increasingly known in Drosophila, where a good match between receptor protein, glomerular identity, and projection neuron innervation pattern is found across individuals. Assume that we cut through the tract of axons connecting the antennal lobe to the mushroom bodies during odor stimulation: unless we know the identity of each axon, we will never be able to deduce the odor responsible for that pattern. Neither relative spatial position nor temporal patterns can help. On the other hand, if we know the activity pattern of olfactory glomeruli, with their identity, we can deduce the odor regardless of their relative spatial arrangement. Clearly, the olfactory code requires an identity component. However, even though the identity information may be sufficient, for an external observer, for the still limited odor/glomeruli space explored, we cannot exclude the possibility that the brain may need additional spatial and/or temporal features. Each insect glomerulus is innervated by 3-5 projection neurons, and in some cases these projection neurons leave the antennal lobe via different tracts. Whether these are redundant or have different properties is unknown. Electrophysiological recordings of honeybees' projection neurons suggest that projection neurons from different morphological tracts afford different processing properties (Müller et al. 2002). An identity code across glomeruli is necessary and potentially sufficient.

\section{Spatial Coding}

As pointed out by Laurent (1999), an identity code does not imply a spatial code. Is the spatial pattern of activity relevant for the code? Presumably, within the 
axon bundle to the mushroom bodies, it is not. However, efficient processing within the antennal lobe may depend on the spatial position of olfactory glomeruli. This view has been strongly advocated for the mammalian olfactory bulb, where local neurons may create a center-surround inhibitory network (Aungst et al. 2003). In many brain areas, convenient spatial arrangement optimizes processing and/or total wiring length (e.g., in the visual system; Durbin and Mitchison 1990). Spatial arrangements also produce a topology: In the primary visual cortex, several representation maps are overlaid in a way that has often been shown to be optimal (a retinotopic map, an ocular-dominance map, an orientation and movement map, and a color-coding map are all superimposed in two dimensions). For a multidimensional olfactory world, two dimensions are not sufficient. Therefore, neighborhood relationships may be useful for subgroups of glomeruli but need not be a general rule. For example, in the honeybee, the neighboring glomeruli T1-33, T1-17, and T1-28 form a continuum in the representation of alcohols from 1-nonanol to 1-hexanol, with continuously decreasing carbon chain length (Galizia and Menzel 2001). Still, these odors also activate glomeruli that do not have neighbors with similar response profiles. A look at the morphology of local interneurons strengthens this point: Cell bodies are clustered together, and the main dendrite travels toward the center of the antennal lobe. From this position, all glomeruli are approximately equidistant. There is only little gain in innervating direct neighbors as compared to some more distant glomeruli in such a spherical arrangement. One step further along the processing path, the spatial arrangement of glomeruli in the antennal lobe may also be relevant by influencing the neighborhood relationships of projection neuron target areas in the mushroom bodies and lateral protocerebrum. Indeed, projection neurons with similar axon projection patterns in the lateral protocerebrum tend to receive input from neighboring glomeruli in Drosophila, suggesting that the organization of the lateral protocerebrum mirrors aspects of the organization in the antennal lobe (Marin et al. 2002; Tanaka et al. 2004). The functional relevance of such a map still needs to be proven.

In summary, while possibly used in subgroups of glomeruli, spatial position of insect glomeruli may generally be dictated more by developmental and/or genetic factors, rather than computational constraints. The spatial position of a glomerulus may even be a necessary component for accurate development of the antennal lobe. We note, however that these are two different aspects; if the identity of a neuron is developmentally characterized by its spatial position (e.g., along a gradient, or by molecular markers), then "space" is a necessary variable for a correct wiring of the network. In the fully developed system, this does not mean that "space" is used for computing the olfactory code. Therefore, from a computational point of view, spatial coding in the adult antennal lobe is not necessary, let alone sufficient. 


\section{Temporal Coding: Slow}

Odor-evoked activity patterns evolve over time within one stimulus application, and projection neurons differ with respect to their temporal response properties (Müller et al. 2002). It is not clear whether part of this phenomenon already originates in the OSN responses, which also show complex dynamics in their activity (see above). Within the antennal lobe or olfactory bulb, this slow evolution ameliorates odor representation, leading to a clearer distinction of odors (Galizia et al. 2000; Friedrich and Laurent 2001). How much of this process can help the animal make better decisions? Among the peculiarities of the olfactory system is the recent finding that information about an odor stimulus does not increase with time, but is rather processed in fixed time units (Ditzen et al. 2003; Uchida and Mainen 2003). In these experiments, the time that an animal takes to reach a decision about a previously learned odor against a similar one was measured. Irrespective of odor similarity (and thus task difficulty), the time needed was constant: $690 \mathrm{~ms}$ for honeybees and about $200 \mathrm{~ms}$ for mice (this included the time needed for olfactory recognition as well as motor responses and physical displacement of the animal). Physiological studies of projection neuron responses show that 200-300 ms in locusts (Stopfer et al. 2003) and $400 \mathrm{~ms}$ in bees (Sachse and Galizia 2003) are needed to reach the most distinct odor classification in the antennal lobe, irrespective of odor concentration. Whether the brain reads the sequence of activities up to that point, or takes a snapshot of the optimized representation, remains to be addressed experimentally. The onset time of a stimulus is probably encoded by the homogeneous local interneurons and multiglomerular projection neurons - neurons that innervate most if not all glomeruli fairly uniformly. Any fluctuation in odor-driven activity that happens after that time span is not relevant for the animal in taking a decision regarding this stimulus. They may, however, still be relevant to the processing of future stimuli (e.g., for repetitive stimuli or aspects involved in memorizing an odor). Since we have already argued that the code has a necessary identity component, slow temporal patterns cannot be sufficient for olfactory coding. They may still be necessary, but as yet we do not have conclusive evidence.

\section{Temporal Coding: Fast}

Fast temporal encoding involves synchrony between spikes and their relationship with oscillations. Laurent (2002) has suggested a feedback loop within the mushroom bodies, formed by the input from the antennal lobes (the projection neurons), the Kenyon cells, and lateral horn inhibitory neurons. This loop would efficiently extract only those action potentials from the projection neuron ensemble code that are in phase with the oscillatory activity, (i.e., only these spikes transmit the information from the antennal lobe to the mushroom bodies). This process is relevant for the transition between projection neurons and Kenyon cells. The question arises, however, whether this mechanism implies that all 
nonsynchronized action potentials in projection neurons are wasted; that is, whether they are not involved in coding the odor at all. Given that firing action potentials are among the most energy-intensive activities of the brain (Attwell and Laughlin 2001), such a waste would appear quite inefficient. Indeed, "surplus" spikes may not be wasted at all: Projection neurons make synaptic output within the antennal lobe, and this output is likely not to be filtered by a fast temporal constraint. Therefore, a mechanism that would extract only a subset of action potentials (which are synchronous with other action potentials) as having an effect in the mushroom bodies does not make the other action potentials a waste of energy. They would still influence olfactory coding within the antennal lobe, and possibly in the lateral protocerebrum. As a result, fast temporal spiking patterns may not be relevant for olfactory processing and optimization within the antennal lobe, even if they may be important outside the antennal lobe, in the reformatting of information between antennal lobe and mushroom body.

The pharmacological agent picrotoxin (PTX) blocks chloride channels such as $\mathrm{GABA}_{\mathrm{A}}$ receptors and thus partially removes the effect of local inhibitory networks. Under its influence, bees fail to discriminate two similar odors, but the distinction of two clearly dissimilar odors is not impaired (Stopfer et al. 1997). At the time of publication, the only known effect of PTX was that it disrupted fast oscillation, and consequently the experiment was taken as evidence that fast oscillation and/or synchrony of action potentials are a necessary component of accurate olfactory coding. We now know that PTX also affects the identity code (Figure 13.4). Some projection neurons that do not normally respond to an odor do so when PTX is applied; others stop responding in honeybees (Sachse and Galizia 2002) as well as in flies (Wilson et al. 2004). Therefore, the loss in olfactory discriminatory power observed under PTX may be a result of the suppressed synchronization or of the changed overall activity across projection neurons. To date, we have no experimental evidence to suggest that fast temporal components are necessary for coding odors, nor do we have evidence to the contrary.

\section{Olfactory Glomeruli Extract Information}

For almost every organism, it is essential to encode different aspects of an olfactory stimulus. This means that the quality of an odor should be coded separately from its intensity. Our visual system, for example, has developed circuits for extracting color information over a wide range of light intensities, so that we can recognize the color of a daisy in sunlight as well as in bright moonlight. Moreover, since odors usually occur in nature as complex blends, the olfactory system needs a specific code for each possible odor combination; it should also be able to analyze single components composing mixtures. Next we discuss theories regarding a multichannel readout strategy by the olfactory system to accomplish these complex requirements. 


\section{Signal-to-Noise Ratio versus Sensitivity}

The antennal lobe must first extract meaningful signals from background noise. However, what constitutes in the antennal lobe the signal or noise? Odor-evoked activity patterns are the signal, while activity that is random is noise. It appears odd, then, to observe that projection neurons have very strong and random spontaneous activity, as shown in many electrophysiological recordings. Such a situation does not exist at all in the input. Although some OSNs are spontaneously active, this activity is not temporally complex. In calcium imaging studies, where only projection neurons were labeled, it was possible to observe frequent bouts of coactive glomerular patterns over long stretches of time (see examples at http://galizia.ucr.edu). An experimenter may not be able to distinguish individual events of spontaneous activity from an odor-evoked pattern. The antennal lobe thus appears to decrease the signal-to-noise ratio rather than increase it. What could be the evolutionary advantage for this?

We propose that this is the price for increasing the antennal lobe's sensitivity to weak odor concentrations. Metaphorically speaking, the antennal lobe is a loaded spring of always almost active projection neurons. On the cellular level, homogeneous local interneurons could accomplish such a task since they have access to all glomeruli and can thus inhibit them all. This feedback loop constantly keeps the projection neurons at firing threshold, so that minimal olfactory stimuli will already elicit an odor-evoked pattern. The mechanism for maintaining the set value at the threshold level is to probe it constantly, which is what we see as spontaneous activity. The cost is a loss in signal-to-noise ratio over time. At first sight, this mechanism may interfere with a reliable representation of odor concentration. However, this is not the case. What interferes with the representation of odor concentration is odor adaptation at the periphery, a phenomenon known from all sensory systems. The phasic response properties of OSNs ensure that the olfactory system always measures concentration changes rather than absolute concentrations. In the adapted state, the "loaded spring model" of the antennal lobe ensures that even small increases in odor concentration will lead to them being detected by the animal. The magnitude of this odor concentration increase (i.e., the strength of the olfactory stimulus) remains reliably coded in the response strength across olfactory glomeruli (see below).

There is a similar phenomenon in hearing. Acoustic sensory neurons have physiologically labile mechanical responses, including intensity-dependent nonlinear effects and spontaneous otoacoustic emissions (Hudspeth et al.2000). These mechanisms greatly increase acoustic sensitivity. The spring model that we propose serves the same scope, even though not realized at the level of sensory neurons, but rather centrally with a fast neural network. The pendant of otoacoustic emissions in the auditory system are the spontaneous activity patterns across glomeruli in the olfactory system.

Is there any evidence for this model, apart from the observed spontaneous activity? We think there is currently none, and appropriate experiments should be 
designed. However, there are two experimental findings that would indirectly support this notion, if we add a memory component to the "loaded spring model." One has been published by Stopfer and Laurent (1999): repeatedly giving an odor to a locust increased the signal-to-noise ratio of the resulting activity pattern in the projection neurons, a phenomenon interpreted as being the result of a form of memory within the antennal lobe. This memory may be a shaping of the "loading forces" in the "loaded springs" of the antennal lobe, so that activity corresponding to that odor would preferentially become suprathreshold. Another experiment was performed by R. Galan and M. Weidert (unpublished). In calcium imaging experiments in honeybees, they found that traces of a given odor persist in the patterns of spontaneous activity for several minutes, providing direct evidence of such reshaped "loading forces."

\section{Odor Identity as Combinatorial Patterns}

As noted above, an identity code with individual glomeruli as units is sufficient for odor identification from the activity patterns. Since the input pattern is contrast enhanced and optimized within the antennal lobe network, the output pattern is even more "powerful" in coding an enormous number of distinct activity patterns and thus olfactory stimuli. However, if odor quality is represented by the identity component of the odor responses, it requires concentration-invariant activity patterns over a wide range of odor concentration to ensure correct odor quality recognition. Indeed, calcium imaging responses of projection neurons in honeybees were qualitatively stable over a concentrations range of up to $4 \log$ units (Sachse and Galizia 2003), with stronger odors increasing response intensity without changing the relative pattern across glomeruli. Interestingly, at the input level the activity patterns were quite affected by odor concentration. Thus odor quality coding and concentration invariance is probably achieved by the neural network within the antennal lobe. Heterogeneous local interneurons are most likely responsible for this process: They selectively innervate a limited number of glomeruli and can therefore optimize very specific contrasts of glomerular activity. The identity of an odor could thus be represented in the combination of activated glomeruli and could be relayed by uniglomerular projection neurons to higher processing centers.

The similarity between microcircuits in the retina and the olfactory bulb has been pointed out by Shepherd and Greer (1998). Color coding in the retina is also optimized to code quality as an intensity-invariant entity. In the retina, three types of sensory neurons create a space formed by two color-opponent channels, in which it is not the absolute activity, but rather the relative activity of the input channels, that is extracted. For example, in the human retina, there are two types of color-opponent ganglion cells that send their signals to the thalamus: either red-green or yellow-blue cells. Activity in these cells reflects the color properties of an object, to a large degree independent of its intensity. They are 
calculated by the retina microcircuits as contrasts between sensory neurons. For example, the red-green channel receives antagonistic input from the middlewavelength receptor ("green") and the long-wavelength receptor ("red"). Consequently, this channel is sometimes called "red-minus-green." The response properties of projection neurons and the innervation patterns of heterogeneous local interneurons are ideally suited for the necessary interactions among glomeruli. However, based on the multidimensional olfactory input space, we would expect a multidimensional odor-opponency output space with possibly quite complex response properties. What are the chances of there being a vanilla-minus-garlic-channel? This is an area where the lack in psychophysical knowledge is blatant. With the possible exception of some pheromone systems, there are as yet no documented examples of odor-opponency channels.

\section{Odor Component Analysis}

Most naturally occurring odors are complex blends of a large number of volatile compounds. Additionally, the turbulent nature of air leads to odor plumes being mixed in a chaotic fashion. This creates a conundrum for the olfactory system: synthetic representations of the blend or an analytical separation of the components. To investigate this issue, the responses to odor mixtures must be analyzed. Several studies have asked how many odors animals can differentiate in a mixture. However, the results cannot be generalized: Every perfumer knows that some odors blend, while others do not; thus the number of discriminable odors depends on the quality of the odors mixed. Electrophysiological studies have shown several kinds of mixture interactions. The presence of an odor B can interfere with the normally strong response to an odor A, which is termed mixture suppression. Conversely, a neuron or glomerulus may respond to a binary mixture with a response that exceeds the summed responses to the single components, defined as synergism. An inhibitory network within the antennal lobe geared at sharpening odor-response patterns should have the effect of creating stronger mixture interactions for mixtures of similar odors than for odors with completely different response patterns. Indeed, calcium imaging responses from honeybee projection neurons to binary mixtures showed inhibitory mixture interactions only for odors that are chemically closely related, such as alcohols which only slightly differed in carbon chain length. This effect was odor and glomerulus specific and could be modified pharmacologically, suggesting that heterogeneous local interneurons are the primary mediators of this effect (Sachse and Galizia, unpublished). Psychophysically, this means that similar odors in a mixture "compose" a new odor, which should make it difficult for the olfactory system to extract the single odor components (synthetic representation), while mixtures of dissimilar substances are represented as the sum of the optimized representation of each component (analytical representation). Indeed, behavioral data in rats support this idea by showing that rats perceive 
binary mixtures composed of dissimilar odors as very similar to their components, whereas binary mixtures containing similar odors appeared dissimilar to the animal (Wiltrout et al. 2003). By increasing the number of components in an odor mixture, mixture interactions are likely to increase and further reduce the similarity to the single component patterns. With this coding strategy of odor mixtures, the olfactory system reveals a mechanism that allows representation of each possible odor mixture without saturating the olfactory code but losing analytical information.

\section{Odor Concentration as a Continuous Variable}

Animals can be trained to respond to an odor with a conditioned response (associative conditioning). If an animal is given a novel odor and responds to it, it has generalized from the trained odor to the novel one. Behavioral experiments show that animals generalize within the same odor over a wide range of concentrations (suggesting a concentration-invariant code) (Borst 1983) or only towards higher concentrations (Pelz et al. 1997). Other experiments have shown that bees can learn to distinguish tenfold concentrations from another, if sufficiently trained. This argues for the maintenance of the concentration information, albeit in a way that needs more training to be learned (Ditzen et al. 2003). Since the spatial activity patterns are widely concentration invariant, the intensity is unlikely to be coded in the spatial combination of activated cells/ glomeruli. However, total activity intensity in the antennal lobe increases continuously with rising odor concentration (Sachse and Galizia 2003). In honeybees and flies, this information would be accessible for multiglomerular projection neurons. These neurons send their axons to the lateral protocerebrum (unlike the uniglomerular projection neurons, which innervate both the lateral protocerebrum and the mushroom bodies). Interestingly, at least parts of these neurons are GABAergic, which means that with increasing odor concentration they would inhibit increasingly their target neurons. Thus the coding of quality and intensity might already be separated by different neuron types in the honeybee antennal lobe and, later, even separated and processed in different brain structures.

Electrophysiological responses of projection neurons in locusts to different odor concentrations reveal a different situation (Stopfer et al. 2003). In these experiments, the response of a single projection neuron to different odor concentrations appeared unpredictable and did not correlate with odor intensity. Thus, each odor at each concentration had its own representation that seemed to be unrelated to that of the same odor at other concentrations. This is different from the continuous representation observed in honeybees. This difference might have morphological reasons: data in the honeybee were recorded from uniglomerular projection neurons, while locust projection neurons collect information from many glomeruli (Figure 13.2). Despite the striking difference in the responses to 
odor concentrations in the two studies, the resulting representation of each odor in a multidimensional space either defined by the activated glomeruli (honeybee) or by the dynamics of different projection neurons (locust) is remarkably similar. In both cases the representation of odor quality is clearly separated into different clusters, whereas odor intensity appears in a continuous order within each quality cluster. Both studies agree on a separate representation of quality versus a continuous coding of intensity, even though the analysis of olfactory responses measured in the two species produced completely different response properties.

It is unknown how odor intensity is treated in the reformatting step between projection neurons and Kenyon cells. For example, in the responses to a series of different odor concentrations, the representation in the mushroom body lips may show some degree of concentration invariance. Our results from the antennal lobe allow for such a mechanism, since the relative activity of glomeruli is quite stable over several orders of magnitude (see above). Alternatively, as has been previously suggested (Heisenberg 2003), the increased overall activity found in the antennal lobe may lead to more Kenyon cells being activated.

\section{Further Sharpening by Synchrony?}

We have found that the antennal lobe optimizes odor representation, as described above (Sachse and Galizia 2002, 2003). A mathematical analysis of spatial activity patterns resulted in the best odor representation if only the strongest glomeruli were taken into account. This suggests that odor representation could even be improved if higher-order brain centers were to read out just the strongest projection neuron responses, raising the question of what mechanism could accomplish such a selective extraction of a subpattern. As mentioned earlier, coactivated projection neurons tend to synchronize in locusts due to the distributed action of GABAergic local interneurons. It is conceivable that projection neurons from strongly activated glomeruli may synchronize more than those from weakly activated ones. If the synchronous projection neuron spikes were the only information affecting higher-order brain centers, the olfactory system would have evolved a strategy for a selective readout. Nonsynchronous projection neuron spikes are probably not useless and are involved in processing mechanisms within the antennal lobe (see above) and/or in conveying information to brain areas other than the mushroom bodies, such as the lateral protocerebrum. An even simpler solution would exploit the statistics of projection neuron responses. Instantaneous firing frequencies of projection neurons go well into the hundreds of spikes/s. These neurons will always contribute with a spike in every readout epoch. Such a mechanism would create an efficient filter without the need of a dedicated network within the antennal lobe. Future experiments are necessary to elucidate the relationship of spatial and temporal features of olfactory responses. 


\section{PROPOSITIONS}

Glomerular microcircuits are made up of a variety of morphologically distinct cells. We propose that processing is a property of the network. Attributing "tasks" to each cell type can only result in a caricature of the system's operation. Nevertheless, such simplifications aid an understanding of the basic mechanisms, and thus we reformulate the thoughts expressed above in terms of their most prominent cellular components.

1. Gain setting: Homogeneous local interneurons. Homogeneous local interneurons innervate the antennal lobe globally. They set the background activity close to the threshold, thus optimizing the system's sensitivity and preventing an overloading of the system.

2a. Contrast enhancement: Heterogeneous local interneurons. Heterogeneous local interneurons create contrasts between a single, densely innervated glomerulus (synaptic input) and a limited number of diffusely innervated glomeruli (synaptic output). They reduce the correlation between glomerular responses in a glomerulus-specific way, perhaps creating odor-opponency circuits.

2b. Odor mixture interactions: Heterogeneous local interneurons. One consequence is that similar odors blend in a way that makes extraction of component identity difficult (synthetic representation), while mixtures of dissimilar odors can still be analyzed (analytical representation).

3. Continuous concentration readout: Multiglomerular projection neurons. Multiglomerular projection neurons have access to the entire antennal lobe. They may respond to global activity, and thus give information about stimulus timing (onset/offset) and concentration.

4. Selective readout: Uniglomerular projection neurons. Uniglomerular projection neurons extract the activity in each glomerular microcircuit and project to higher-order brain centers. The identity of an odor is encoded in the combinatorial activity pattern across their identified axons.

\section{ACKNOWLEDGMENTS}

We thank Kenta Asahina, Richard Benton, Andreas Keller, Mattias Larsson, Matthieu Louis, Philipp Peele, Daniela Pelz, Ana F. Silbering, and Leslie B. Vosshall for critical comments to the manuscript.

\section{REFERENCES}

Abel, R. 1997. Das olfaktorische System der Honigbiene: Elektrophysiologische und morphologische Charakterisierung von Antennallobusneuronen und deren Beteiligung beim olfaktorischen Lernen. Ph.D. Thesis. Berlin: Freie Universität Berlin. 
Attwell, D., and S.B. Laughlin. 2001. An energy budget for signaling in the grey matter of the brain. J. Cereb. Blood Flow Metab. 21:1133-1145.

Aungst, J.L., P.M. Heyward, A.C. Puche et al. 2003. Centre-surround inhibition among olfactory bulb glomeruli. Nature 426:623-629.

Borst, A. 1983. Computation of olfactory signals in Drosophila melanogaster. J. Comp. Physiol. A 152:373-383.

Brockmann, A., and B. Brückner. 1995. Projection pattern of poreplate sensory neurones in honeybee worker Apis mellifera L. Hymenoptera: Apidae. Intl. J. Insect Morphol. Embryol. 24:405-411.

Christensen, T.A., B.R. Waldrop, and J.G. Hildebrand. 1998. Multitasking in the olfactory system: Context-dependent responses to odors reveal dual GABA-regulated coding mechanisms in single olfactory projection neurons. J. Neurosci. 18:59996008 .

de Bruyne, M., K. Foster, and J.R. Carlson. 2001. Odor coding in the Drosophila antenna. Neuron 30:537-552.

Ditzen, M., J.F. Evers, and C.G. Galizia. 2003. Odor similarity does not influence the time needed for odor processing. Chem. Senses 28:781-789.

Durbin, R., and G. Mitchison. 1990. Adimension reduction framework for understanding cortical maps. Nature 343:644-647.

Fiala, A., T. Spall, S. Diegelmann et al. 2002. Genetically expressed cameleon in Drosophila melanogaster is used to visualize olfactory information in projection neurons. Curr. Biol. 12:1877-1884.

Fonta, C., X.J. Sun, and C. Masson. 1993. Morphology and spatial distribution of bee antennal lobe interneurones responsive to odours. Chem. Senses 18:101-119.

Friedrich, R.W., and G. Laurent. 2001. Dynamic optimization of odor representations by slow temporal patterning of mitral cell activity. Science 291:889-894.

Galizia, C.G., and B. Kimmerle. 2004. Physiological and morphological characterization of honeybee olfactory neurons combining electrophysiology, calcium imaging, and confocal microscopy. J. Comp. Physiol. A. 190:21-38.

Galizia, C.G., A. Küttner, J. Joerges, and R. Menzel. 2000. Odour representation in honeybee olfactory glomeruli shows slow temporal dynamics: An optical recording study using a voltage-sensitive dye. J. Insect Physiol. 46:877-886.

Galizia, C.G., and R. Menzel. 2001. The role of glomeruli in the neural representation of odours: Results from optical recording studies. J. Insect Physiol. 47:115-130.

Hansson, B.S., and T.A. Christensen. 1999. Functional characteristics of the antennal lobe. In: Insect Olfaction, ed. B.S. Hansson, pp. 125-161. New York: Springer.

Heisenberg, M. 2003. Mushroom body memoir: From maps to models. Nat. Rev. Neurosci.4:266-275.

Hildebrand, J.G., and G.M. Shepherd. 1997. Mechanisms of olfactory discrimination: Converging evidence for common principles across phyla. Ann. Rev. Neurosci. 20:595-631.

Homberg, U., and U. Müller. 1999. Neuroactive substances in the antennal lobe. In: Insect Olfaction, ed. B.S. Hansson, pp. 181-206. New York: Springer.

Hudspeth, A.J., Y. Choe, A.D. Mehta, and P. Martin. 2000. Putting ion channels to work: Mechanoelectrical transduction, adaptation, and amplification by hair cells. PNAS 97:11,765-11,772.

Laurent, G. 1999. A systems perspective on early olfactory coding. Science 286: $723-728$. 
Laurent, G. 2002. Olfactory network dynamics and the coding of multidimensional signals. Nat. Rev. Neurosci. 3:884-895.

Marin, E.C., G.S.X.E. Jefferis, T. Komiyama, H. Zhu, and L. Luo. 2002. Representation of the glomerular olfactory map in the Drosophila brain. Cell 109:243-255.

Müller, D., R. Abel, R. Brandt, M. Zöckler, and R. Menzel. 2002. Differential parallel processing of olfactory information in the honeybee, Apis mellifera L. J. Comp. Physiol. A 188:359-370.

Nässel, D.R. 1999. Histamine in the brain of insects: A review. Microsc. Res. Tech. 44:121-136.

$\mathrm{Ng}$, M., R.D. Roorda, S.Q. Lima et al. 2002. Transmission of olfactory information between three populations of neurons in the antennal lobe of the fly. Neuron 36: 463-474.

Pelz, C., B. Gerber, and R. Menzel. 1997. Odorant intensity as a determinant for olfactory conditioning in honeybees: Roles in discrimination, overshadowing and memory consolidation. J. Exp. Biol. 200 (Pt 4):837-847.

Perez-Orive, J., O. Mazor, G.C. Turner et al. 2002. Oscillations and sparsening of odor representations in the mushroom body. Science 297:359-365.

Sachse, S., and C.G. Galizia. 2002. Role of inhibition for temporal and spatial odor representation in olfactory output neurons: A calcium imaging study. J. Neurophysiol. 87:1106-1117.

Sachse, S., and C.G. Galizia. 2003. The coding of odour-intensity in the honeybee antennal lobe: Local computation optimizes odour representation. Eur. J. Neurosci. 18:2119-2132.

Shepherd, G.M., and C.A. Greer. 1998. Olfactory bulb. In: The Synaptic Organization of the Brain, ed. G.M. Shepherd, pp. 159-203. New York: Oxford Univ. Press.

Stopfer, M., S. Bhagavan, B.H. Smith, and G. Laurent. 1997. Impaired odour discrimination on desynchronization of odour-encoding neural assemblies. Nature 390:70-74.

Stopfer, M., V. Jayaraman, and G. Laurent. 2003. Intensity versus identity coding in an olfactory system. Neuron 39:991-1004.

Stopfer, M., and G. Laurent. 1999. Short-term memory in olfactory network dynamics. Nature 402:664-8.

Tanaka, N.K., T. Awasaki, T. Shimada, and K. Ito. 2004. Integration of chemosensory pathways in the Drosophila second-order olfactory centers. Curr. Biol. 14:449-457.

Uchida, N., and Z.F. Mainen. 2003. Speed and accuracy of olfactory discrimination in the rat. Nat. Neurosci. 6:1224-1229.

Vosshall, L.B. 2001. The molecular logic of olfaction in Drosophila. Chem. Senses 26:207-213.

Wang, J.W., A.M. Wong, J. Flores, L.B. Vosshall, and R. Axel. 2003. Two-photon calcium imaging reveals an odor-evoked map of activity in the fly brain. Cell 112: 271-282.

Wilson, R.I., G.C. Turner, and G. Laurent. 2004. Transformation of olfactory representations in the Drosophila antennal lobe. Science 303:366-370.

Wiltrout, C., S. Dogra, and C. Linster. 2003. Configurational and nonconfigurational interactions between odorants in binary mixtures. Behav. Neurosci. 117:236-245. 